“C 2017 IEEE. Personal use of this material is permitted. Permission from IEEE must be obtained for all other uses, in any current or future media, including reprinting/republishing this material for advertising or promotional purposes, creating new collective works, for resale or redistribution to servers or lists, or reuse of any copyrighted component of this work in other works." 


\title{
Compact, Frequency-Reconfigurable Filtenna with Sharply Defined Wideband and Continuously Tunable Narrowband States
}

\author{
Ming-Chun Tang, Senior Member, IEEE, Zheng Wen, Hao Wang, Student Member, IEEE, Mei Li, \\ Member, IEEE, and Richard W. Ziolkowski, Fellow, IEEE
}

\begin{abstract}
A compact, frequency-reconfigurable filtenna with sharp out-of-band rejection in both its wideband and continuously tunable narrowband states is presented. It is intended for use in cognitive radio applications. The wideband state is the sensing state and operationally covers $2.35-4.98 \mathrm{GHz}$. The narrow band states are intended to cover communications within the $3.05 \mathrm{GHz}$ to $4.39 \mathrm{GHz}$ range, which completely covers the WiMAX band and the satellite communications C-band. A PIN diode is employed to switch between these wide and narrow band operational states. Two varactor diodes are used to shift the operational frequencies continuously among the narrowband states. The filtenna consists of a funnel-shaped monopole augmented with a reconfigurable filter; it has a compact electrical size: $0.235 \lambda_{L} \times 0.392 \lambda_{L}$, where the wavelength $\lambda_{L}$ corresponds to the lower bound of its operational frequencies. The measured reflection coefficients, radiation patterns, and realized gains for both operational states are in good agreement with their simulated values.
\end{abstract}

Index Terms - Cognitive radio, filtenna, frequencyreconfigurable, monopole antenna, reconfigurable antennas, reconfigurable filters

\section{INTRODUCTION}

$\mathrm{W}$ ITH the expansive development of wireless communication technologies and applications, the available frequency spectrum is becoming more and more congested. This is particularly true, as noted by the Federal

Manuscript received on Apr. 24, 2017, and revised on Jun. 23, 2017.

This work was supported in part by the National Natural Science Foundation of China contract number 61471072, in part by graduate scientific research and innovation foundation of Chongqing, China contract number CYS16020, in part by the Fundamental Research Funds for the Central Universities contract number 106112015CDJZR165510, in part by China Postdoctoral Science Foundation contract number 2016M590860, in part by Funding of the Young Backbone Teachers in Colleges and Universities of Chongqing contract number 0307001104102 , and in part by the Australian Research Council grant number DP160102219.

M. - C. Tang, Z. Wen, H. Wang and M. Li are with the Key Laboratory of Dependable Service Computing in Cyber Physical Society Ministry of Education, College of Communication Engineering, Chongqing University and also with the Chongqing Engineering Laboratory of High Performance Integrated Circuits, College of Communication Engineering, Chongqing University, Chongqing 400044, China (E-mail: tangmingchun@ @ cqu.edu.cn);

R. W. Ziolkowski is with the University of Technology Sydney, Global Big Data Technologies Centre, Ultimo NSW 2007, Australia, and the Department of Electrical and Computer Engineering, University of Arizona, Tucson, AZ 85721, USA (E-mail: Richard.Ziolkowski@uts.edu.au).
Communications Commission (FCC) [1], because the available frequency spectrum is not utilized efficiently. Cognitive radio (CR) technology has been recently advanced as one of the effective methods to solve this problem. Consequently, it has attracted considerable attention.

A CR system generally possesses the ability to sense the available spectra and then direct communications to be performed over the detected, unoccupied frequencies without impacting other users. Ideally it would consist of one antenna that performs both the sensing and communications functions. Owing to their inherent tunable and dynamically-controlled features, frequency-agile and reconfigurable technologies have become practical approaches to empower both functionalities $[2,3]$. As a result, many years of effort have been devoted to designing reconfigurable antennas for CR applications [4-18].

Two switching approaches are common. On the one hand, PIN diodes or GaAs field effect transistors are incorporated into an antenna to switch between its wideband state, which is used to sense the available spectra, and its multiple, discrete, fixed narrow-band states for communications [4-15]. On the other hand, PIN and varactor diodes are employed, respectively, to switch between the wideband and the narrowband states, and to realize continuous tuning amongst the various narrowband states [16-18].

Unfortunately, these reported antennas have various drawbacks which limit their practical CR applications. The elements which provide the ON/OFF switching functions facilitate only a few, fixed narrow bands. One does not obtain a frequency-agile property which allows one to continuously shift the operational narrow-bands across the entire sensing band [4-15]. In contrast, employing continuously-controllable elements overcomes this issue. Nevertheless, the related frequency-agile bandwidths are quite narrow $(\sim 20 \%)$ and their configurations are not compact $[17,18]$. Moreover, to the best of our knowledge, the CR antennas that have been reported to date do not provide the necessary out-of-band rejection performance characteristics throughout the wideband state [4-18]. It would be advantageous to effectively eliminate the impact of any signals that lie outside of the sensing spectrum, i.e., to have very sharp out-of-band rejection properties associated with the filter defining the sensing spectrum. Furthermore, one would like to reduce or eliminate the interconnection losses that arise in the standard arrangement in which an add-on filter is connected between the source and the antenna. Integrating the filter into the antenna would yield a more compact, simpler, and lower cost CR system [19-21]. 
A compact, frequency-reconfigurable filtenna is detailed in this paper. It has excellent bandpass performance characteristics in both its wideband and continuously tunable narrowband states, making it very attractive for CR applications. In contrast to many of the previously reported reconfigurable CR filtennas $[4-8,17,18]$, it not only has a simple and compact configuration, but it also possesses sharp out-of-band rejection properties in both its wideband and narrowband states. The optimized reconfigurable filter design is first introduced in Section II, and its performance characteristics are analyzed. In Section III, this compact filter is seamlessly integrated into a wideband funnel-shaped monopole antenna to achieve the frequency-reconfigurable filtenna. The fabrication and testing of a prototype of the optimized design is described. The simulated and measured results are compared. They are in good agreement, confirming the design strategy. Furthermore, it is demonstrated that with the frequency agility attained through its composite filter section, the filtenna possesses two excellent reconfigurability advantages. First, the switch between its wideband and narrowband operational states is realized simply and effectively with a single PIN diode. Second, it is demonstrated that its narrowband operational frequencies can be continuously and easily shifted from 3.05 to $4.39 \mathrm{GHz}$ using just two varactors, yielding a $36.0 \%$ frequency-agile fractional bandwidth. This frequency range covers entirely both the WiMAX band $(3.3-3.6 \mathrm{GHz})$ and the satellite communications C-band (3.7-4.2 GHz). Very sharp out-of-band rejection performance is reported in both operational states. The size of this optimized filtenna design is only $0.235 \lambda_{L} \times 0.392 \lambda_{L}$, where $\lambda_{L}=\mathrm{c} / f_{L}$ is the wavelength corresponding to the lowest frequency $f_{L}$ of its entire $-10 \mathrm{~dB}$ operational frequency range. A direct comparison of the performance characteristics of the filtenna with those of the CR antennas reported in [4-18] is given; it establishes the fact that this filtenna is a simpler and more compact design. Moreover, its $36 \%$ frequency-agile fractional bandwidth is shown to be wider than the previously reported cases [17, 18] without degrading the realized gain values. Finally, some conclusions are drawn in Section IV.

We note that all of the metallic elements modeled in the filter and antenna designs were chosen to be copper with its known material parameters: $\varepsilon_{r}=1.0, \mu_{r}=0.999991$ and bulk conductivity $\sigma=5.8 \times 10^{7} \mathrm{Siemens} / \mathrm{m}$. All of the numerical simulations and their optimizations were performed using the frequency domain, finite element-based ANSYS/ANSOFT High Frequency Structure Simulator (HFSS), version 13.0 [22].

\section{RECONFIGURABLE COMPOSITE FILTER DESIGN}

A compact frequency-reconfigurable filter was developed based on the wideband, bandpass filter design methodology given in $[23,24]$. The composite design is depicted in Fig. 2. It was engineered to be realized simply with standard, printed circuit board (PCB) technology. The copper traces are 0.017 $\mathrm{mm}$ thick $(1 / 2 \mathrm{oz})$ and are etched on both sides of a Rogers 4350B substrate, whose relative dielectric constant $\varepsilon_{r}=3.48$, relative permeability $\mu_{\mathrm{r}}=1.0$, and loss tangent $\tan \delta=0.0038$. The optimized design parameters are listed in Table I. The composite filter design is composed mainly of a C-shaped narrowband resonator [23], an E-shaped wideband resonator
[24], two parallel-coupled transmission lines, and a 50-ohm input/output microstrip line. The E-shaped wideband resonator is located halfway between the input and output ports. It is connected to a microstrip line that is capacitively coupled to the 50-ohm microstrip transmission line that connects the input and output ports and is directly connected to the ground via a PIN diode integrated with (metallic) Via . $_{1}$

The equivalent circuit of the E-shaped resonator is shown in Fig. 1, emphasizing its coupling features. According to previous theoretical analyses and experiments [25-27], this structure has the same performance as a filter with capacitive terminations. Consequently, two transmission zeros are introduced into its lower and upper stopbands, which improve the selectivity of the filter.

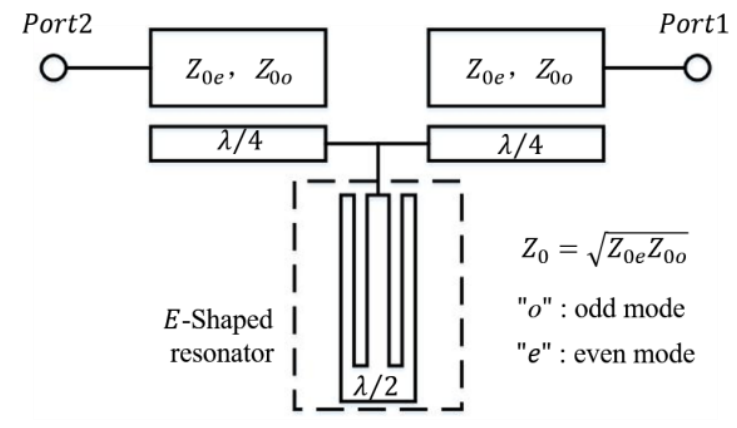

Fig. 1 Coupling schematic of the E-shaped wideband resonator.

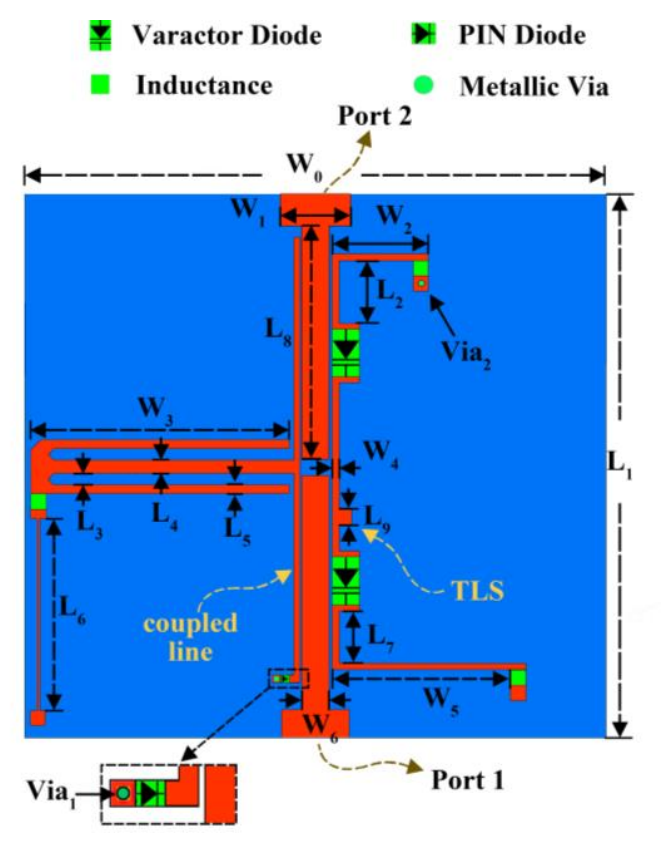

Fig. 2 Geometry of the frequency-reconfigurable composite filter design.

The PIN diode is placed at the end of this coupled line close to the Port 1. The M/A-COM MA4GP907 PIN diode was selected. According to its datasheet, it acts approximately like a $4 \mathrm{ohm}$ resistor in its ON state and as a $0.025 \mathrm{pF}$ capacitor in its OFF state [28]. The DC bias line for this diode is positioned at the bottom left side of Fig. 2and is connected to one end of a RF-blocking $56 \mathrm{nH}$ coil inductor, whose other end is connected the left side of the E-shaped resonator. This position minimizes its influence on the filter performance. 
TABLE I.

THE OPTIMIZED DESIGN PARAMETERS OF THE FREQUENCY-RECONFIGURABLE FILTER (ALl DiMENSIONS ARE IN MILLIMETERS)

\begin{tabular}{|c|c|c|c|c|}
\hline$L_{1}=28$ & $L_{2}=3.3$ & $L_{3}=0.6$ & $L_{4}=0.7$ & $L_{5}=0.45$ \\
\hline$L_{6}=10$ & $L_{7}=2.8$ & $L_{8}=12.1$ & $L_{9}=0.8$ & $W_{0}=30$ \\
\hline$W_{1}=3.6$ & $W_{2}=5$ & $W_{3}=13.41$ & $W_{4}=0.32$ & $W_{5}=9.2$ \\
\hline$W_{6}=1.4$ & \multicolumn{4}{|c|}{$N$ ull } \\
\hline
\end{tabular}

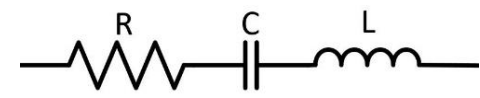

Fig. 3 Equivalent circuit of the varactor diode.

The C-shaped narrowband resonator is located on the right side of the input/output line, $0.14 \mathrm{~mm}$ from it. One of its ends is also connected to the ground through a $56 \mathrm{nH}$ inductor integrated with a (metallic) via $\left(\mathrm{Via}_{2}\right)$. The other end, near Port 1 , is connected to a DC bias pad through another $56 \mathrm{nH}$ inductor. A varactor diode is inserted into both halves of the coupled-line sections of this C-shaped resonator. The Aerofelx Metellics MGV 125-20-E28 varactor diode was selected for this purpose. According to its datasheet, it can be modeled as the series RLC equivalent circuit illustrated in Fig. 3 [29]. The equivalent circuit is composed of a series resistance (R), a parasitic inductance (L), and a total capacitance (C), which consists of the junction capacitance $\left(\mathrm{C}_{\mathrm{j}}\right)$ and a parasitic capacitance $\left(\mathrm{C}_{\mathrm{p}}\right)$. The values of $\mathrm{R}, \mathrm{L}$, and $\mathrm{C}$ are, respectively, $1.75 \mathrm{ohm}, 0.4 \mathrm{nH}$, and $0.08 \mathrm{pF}$. The tuning reverse bias voltage of the diode can be varied from 20.0 to $2.0 \mathrm{~V}$, which changes $\mathrm{C}_{\mathrm{j}}$ in the range from 0.1 to $1.0 \mathrm{pF}$.

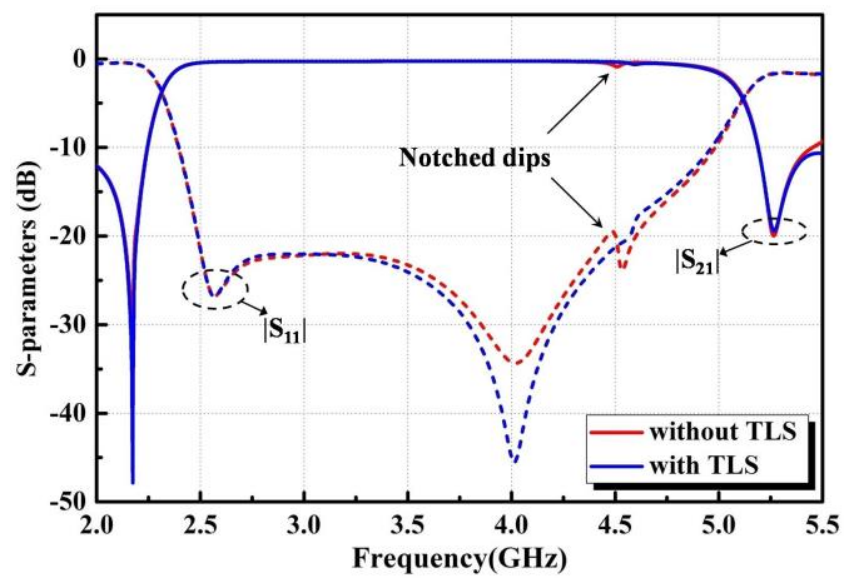

Fig. 4 Simulated S-parameters of the composite filter in its wideband state with and without a square tapered line structure (TLS).

When the PIN diode is turned OFF, the composite filter operates in its wideband state. For a better impedance match to the source in this OFF-state, the effective capacitance of each varactor diode is adjusted to be $0.1 \mathrm{pF}$. In contrast, when the PIN diode is turned ON, the composite filter operates in its narrowband states. In this case, the power reaching port 2 from port 1 is transmitted mainly through the C-shaped narrowband resonator. Then by varying the voltages applied to the two varactor diodes, one can continuously shift the filter's narrowband operational frequencies across the entire spectrum of the wideband state.
The HFSS-predicted S-parameters of the reconfigurable composite filter design in its wideband state are presented in Fig. 4. They show the filter provides a passband with $\left|S_{11}\right|<-10$ $\mathrm{dB}$ from 2.4 to $5.0 \mathrm{GHz}$. The insertion loss is less than $1 \mathrm{~dB}$ over this passband. Two transmission zeros with the attenuation levels $\sim 47.85 \mathrm{~dB}$ and $\sim 19.43 \mathrm{~dB}$ are, respectively, located at 2.2 $\mathrm{GHz}$ and at $5.3 \mathrm{GHz}$, near the low and high cut-off frequencies. As a result, an excellent out-of-band rejection characteristic is obtained.

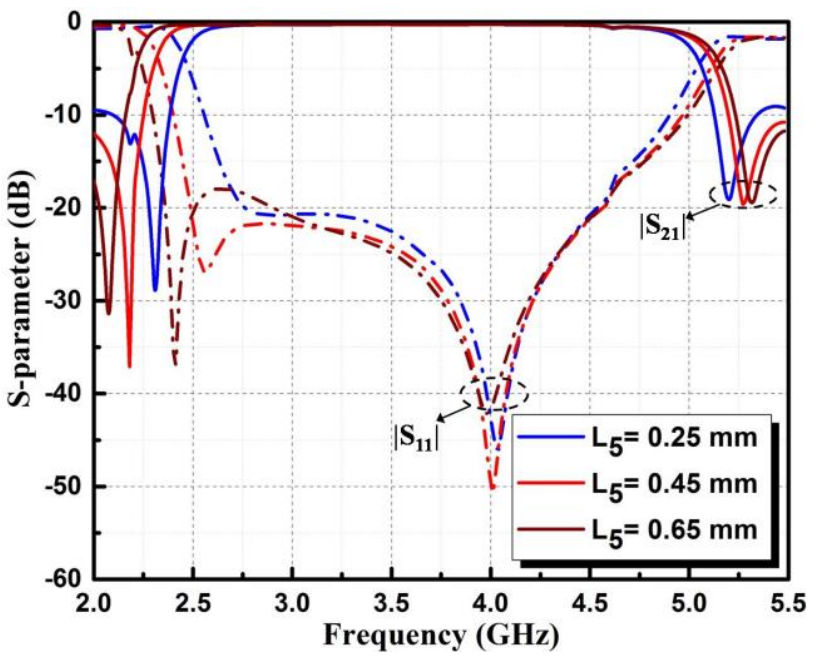

Fig. 5 Impact of the E-shaped structure's stub width, $\mathrm{L}_{5}$, on the composite filter's impedance matching bandwidth.

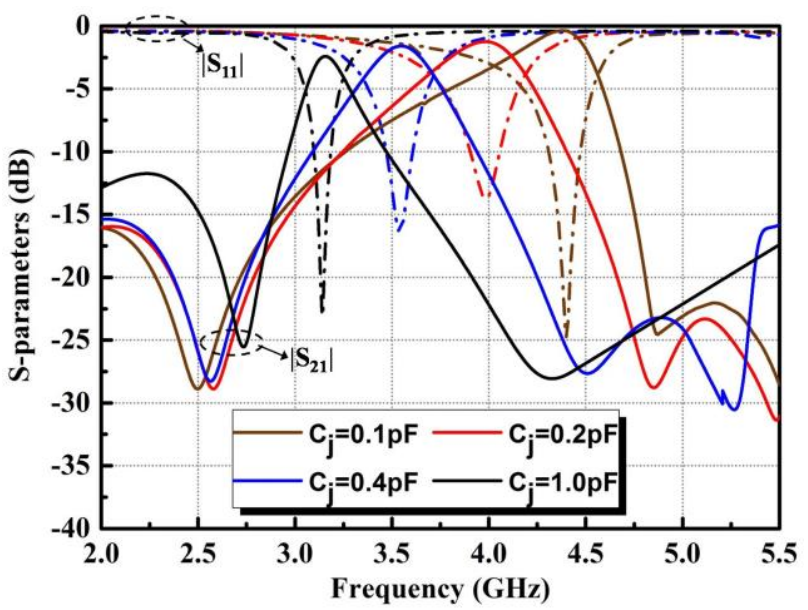

Fig. 6 Impact of the junction capacitance $C_{j}$ of each varactor in the $C$-shaped resonator on the composite filter's performance in its narrowband state.

Note that not all of the input power is transmitted to the output port via the E-shaped resonator when the composite filter is in its wideband state because of the presence of the $\mathrm{C}$-shaped resonator. A fraction of the power passes through it. As Fig. 4 shows, a notched dip appears near $4.5 \mathrm{GHz}$ where the $\mathrm{C}$-shaped resonator becomes self-resonant [30]. In order to eliminate this notched dip within the passband, the square tapered line structure (TLS) shown in Fig. 2is utilized. Its width and length are the same: $\mathrm{L}_{9}=0.8 \mathrm{~mm}$. It is located at the midpoint between the center of the input/output transmission line and the source-side varactor diode. The simulated results with and without the TLS indicate that its presence eliminates 
the notched dip and thus makes the transmission performance more stable across the entire passband.

The simulated S-parameters of the composite filter in its wideband and narrowband states are illustrated, respectively, in Figs. 4 and 5. The impact of one key design parameter, the width of the E-shaped structure's stub, $\mathrm{L}_{5}$, on the transmission performance in the wideband state, is given in Fig. 5. It is readily observed that when $L_{5}$ is increased from 0.25 to 0.65 $\mathrm{mm}$, the passband expands from the range 2.39 to $5.13 \mathrm{GHz}$ (fractional bandwidth $72.87 \%$ ) to the range 2.17 to $5.23 \mathrm{GHz}$ $(82.7 \%)$. Distinctly, $\mathrm{L}_{5}$ controls the lower and upper bounds of the passband.

Fig. 6 shows that by varying the effective capacitance value from 1.0 to $0.1 \mathrm{pF}$, the frequency-agile $-10-\mathrm{dB}$ bandwidth covers the range from 3.09 to $4.47 \mathrm{GHz}$. This means the tunable bandwidth is $1.38 \mathrm{GHz}$, i.e., a $36.51 \%$ fractional bandwidth. Note that our simulation studies have shown that if only one varactor diode was used in the $\mathrm{C}$-shaped resonator, the frequency-agile bandwidth would be reduced by almost half, to the range from 3.15 to $3.86 \mathrm{GHz}$, i.e., the fractional bandwidth would be reduced to $20.26 \%$.

\section{RECONFIGURABLE FILTENNA}

The frequency-reconfigurable filtenna is attained by integrating the compact composite filter into a wideband funnel-shape monopole antenna. The geometry of the structure and photos of the fabricated prototype are shown in Fig. 7. As Figs. 6(a) and 6(b) suggest, the filtenna is constructed using the same substrate. The overall size of this substrate is $\mathrm{L} \times \mathrm{W} \times \mathrm{H}=$ $50 \times 30 \times 1.524 \mathrm{~mm}^{3}$. Accordingly, its total transverse electrical size is $0.235 \lambda_{\mathrm{L}} \times 0.392 \lambda_{\mathrm{L}}$. The optimized design parameters for the frequency-reconfigurable filtenna are given in Table II.

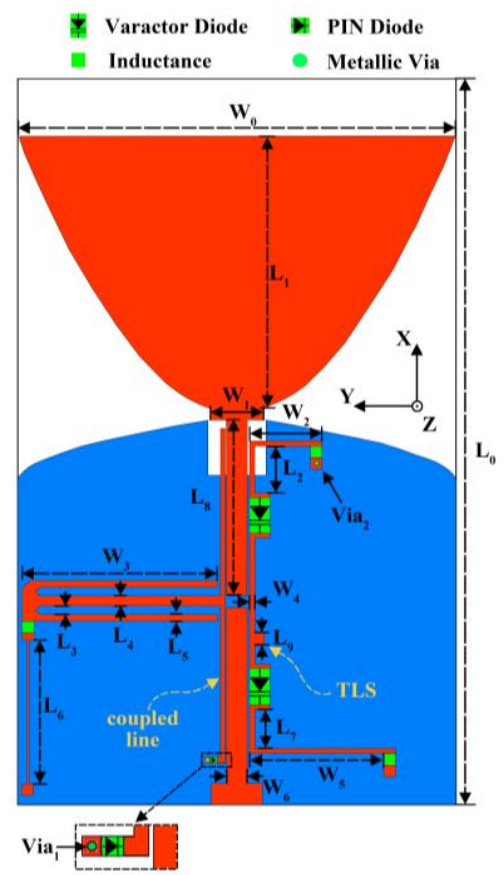

(a)

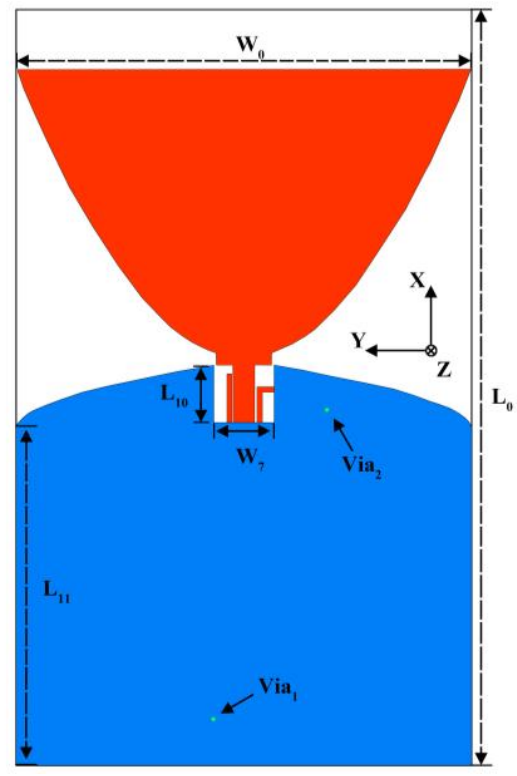

(b)

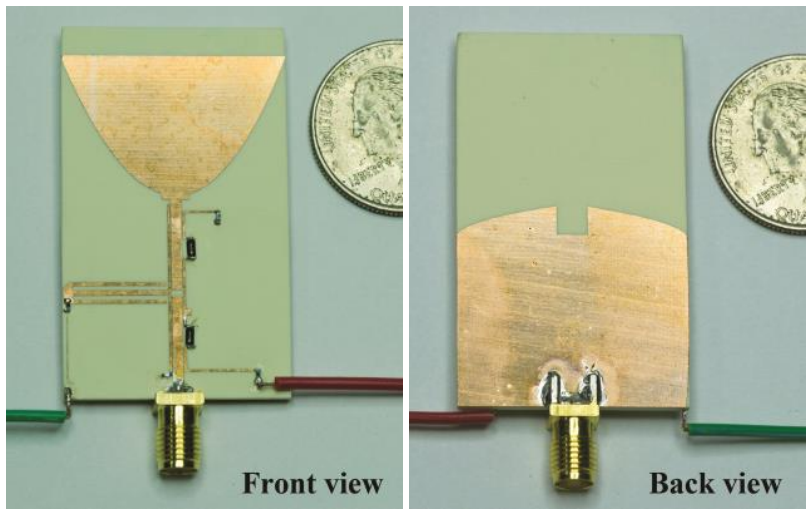

(c)

Fig. 7 Geometry of the frequency-reconfigurable filtenna. (a) Top view, (b) bottom view, and (c) photos of the fabricated prototype.

TABLE II.

THE OPTIMIZED DESIGN PARAMETERS OF THE FREQUENCY-RECONFIGURABLE FILTENNA (ALL DiMENSIONS ARE IN MILLIMETERS)

\begin{tabular}{|c|c|c|c|c|}
\hline$L_{0}=50$ & $L_{1}=18.73$ & $L_{2}=3.3$ & $L_{3}=0.6$ & $L_{4}=0.7$ \\
\hline$L_{5}=0.46$ & $L_{6}=10$ & $L_{7}=2.8$ & $L_{8}=12.1$ & $L_{9}=0.8$ \\
\hline$L_{10}=3.78$ & $L_{11}=22.2$ & $W_{0}=30$ & $W_{1}=3.6$ & $W_{2}=5$ \\
\hline$W_{3}=13.41$ & $W_{4}=0.32$ & $W_{5}=9.2$ & $W_{6}=1.4$ & $W_{7}=4$ \\
\hline
\end{tabular}

The antenna is a funnel-shaped monopole etched on the top side of the substrate board as shown in Fig. 7. The composite filter integrated with the 50-ohm microstrip feed line is directly connected to the base of the funnel. Its shape is designed with the quadratic equations:

$$
\left\{\begin{array}{l}
x=\left(\frac{a}{2}\right) \sin \theta \\
y=b(\cos \theta)^{2}
\end{array}\right.
$$

where here, as shown in Fig. 7(a), a $=W_{0}$ is the width of the substrate and $\mathrm{b}=L_{l}$ is the length of funnel. This quadratic function-based design tailors the input impedance to facilitate 
matching to the $50 \Omega$ source over a broad range of frequencies and produces smooth transitions from one resonant mode to another across that wide bandwidth, while maintaining its compact size [31,32]. The conducting ground plane is etched on the bottom side of the substrate; its configuration is shown in Fig. 7(b). It has a curved upper edge which is also designed with Eqn. (1), but with $a=9.2 \mathrm{~mm}$ and $b=15 \mathrm{~mm}$. A rectangular slot with dimensions $\mathrm{W}_{7} \times \mathrm{L}_{10}=4.0 \mathrm{~mm} \times 3.8 \mathrm{~mm}$ is centered in this curved upper edge. This arrangement also expedites the impedance matching in the wideband state [31, 32]. The optimized filtenna was fabricated and tested; photos of the fabricated prototype are shown in Fig. 7(c).

With its integration with the wideband monopole antenna, the filtenna takes on the characteristics of the composite filter. First, its operation can be switched between the wideband and narrowband states simply by switching the PIN diode to its OFF or ON state, respectively. Second, when it is in its narrowband state, one can continuously shift its operational frequencies amongst its tunable, uniform narrow-band states by simply tuning the bias voltage applied to the two varactors.

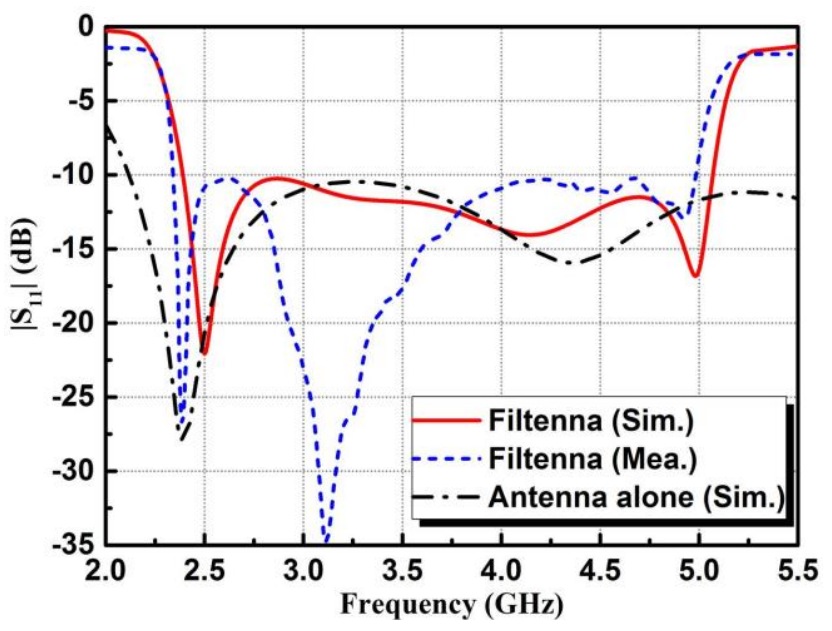

Fig. 8 HFSS-predicted and measured $\left|S_{11}\right|$ values as functions of the source frequency when the filtenna is in its wideband state.

Recall that when a reverse bias voltage is applied to it, the PIN diode is in its OFF state. Then, according to the performance characteristics of the composite filter, the bias voltages applied to each varactor diode should be adjusted to obtain their $0.1 \mathrm{pF}$ junction capacitance states to ensure the best impedance matching. The corresponding simulated and measured S-parameter results for this wideband state of the filtenna are depicted in Fig. 8. The simulated (measured) -10-dB impedance bandwidth is $2.7 \mathrm{GHz}(2.63 \mathrm{GHz})$ from 2.4 (2.35) to $5.1 \mathrm{GHz}(4.98 \mathrm{GHz})$. As a reference, the monopole antenna without the filter section was simulated. These results are also presented in Fig. 8 for easy comparison. The reference has a $7.9 \mathrm{GHz}$ operational bandwidth in the range from 2.10 to 12.0 GHz. As expected [31], the reference antenna response is smooth at both the lower and upper edges of its bandwidth. Comparison with the reference indicates that the filtenna exhibits a sharp cut-off performance at the edges of its operational band. For example, the simulated $\left|S_{11}\right|$ values of the filtenna (reference antenna) are $-2.16 \mathrm{~dB}(-15.56 \mathrm{~dB})$ and -1.79
$\mathrm{dB}(-11.17 \mathrm{~dB})$ at two selected out-of-band frequency points: $2.25 \mathrm{GHz}$ and $5.25 \mathrm{GHz}$, respectively. These results demonstrate that the filtenna has improved rejection levels as high as $13.4 \mathrm{~dB}$ and $9.38 \mathrm{~dB}$, respectively, at these out-of-band frequency points.

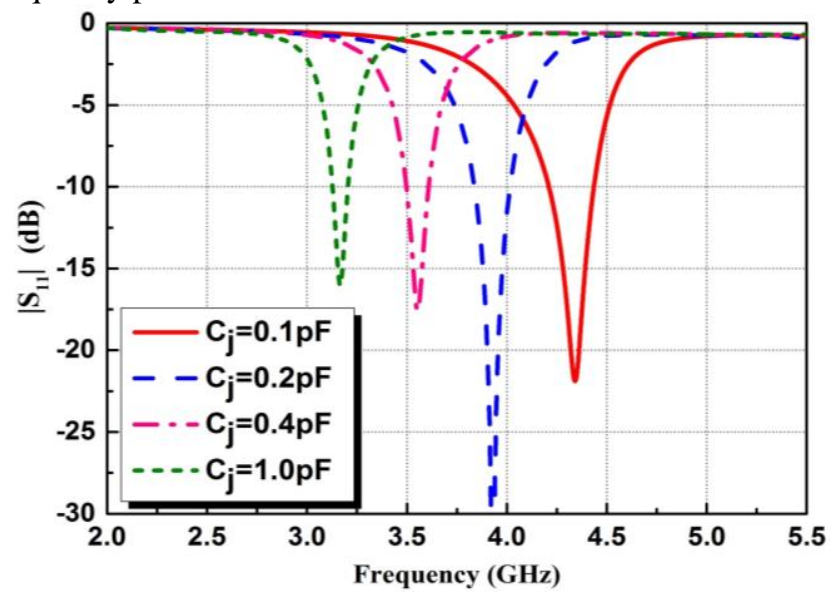

(a)

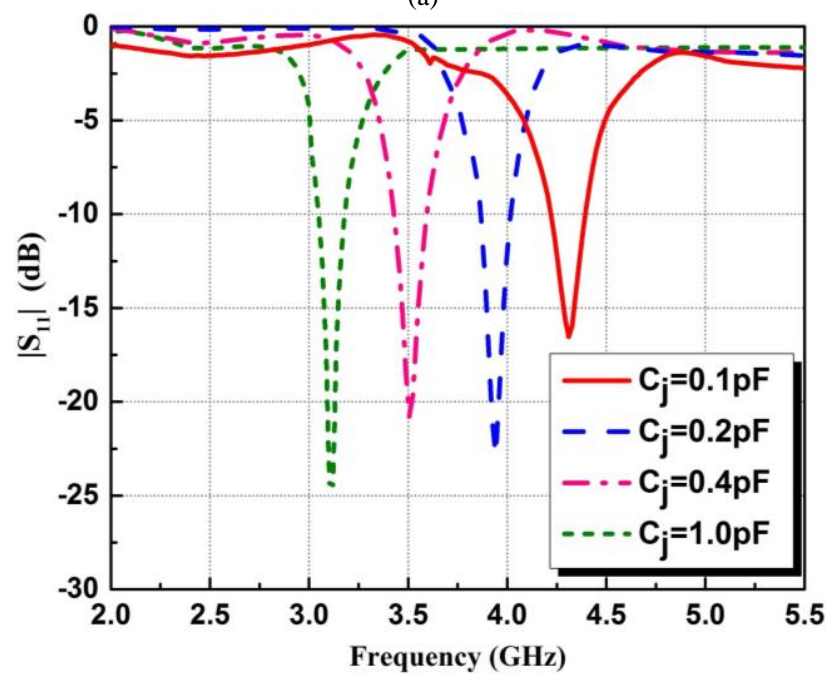

(b)

Fig. 9 HFSS-predicted and measured $\left|S_{11}\right|$ values as functions of the source frequency when the filtenna is in several of its narrowband states; (a) Simulated values, and (b) measured values.

On the other hand, with the forward bias voltage $(1.4 \mathrm{~V})$ applied to it, the PIN diode operates in its ON state. Because the PIN diode acts as a 4-ohm resistor in this case, the coupled line is essentially shorted to the ground and the coupled power resides mainly in the $\mathrm{C}$-shaped narrowband resonator. Recall that this facilitates an effective tuning of the filtenna's narrowband states simply by changing the reverse bias voltage applied to the varactor diodes. Several cases were simulated and measured. The junction capacitance of each varactor diode was set to: $0.1 \mathrm{pF}(20 \mathrm{~V}), 0.2 \mathrm{pF}(9 \mathrm{~V}), 0.4 \mathrm{pF}(4.7 \mathrm{~V})$, and $1 \mathrm{pF}$ $(2 \mathrm{~V})$. As is shown in Fig. 9, the simulated (measured) frequency-agile $\left(\left|\mathrm{S}_{11}\right|<-10 \mathrm{~dB}\right)$ performance of the filtenna in its narrowband state ranges from 3.12 (3.05) to 4.44 (4.39) $\mathrm{GHz}$, i.e., its exhibits as $1.32(1.34) \mathrm{GHz}$ bandwidth. Thus, the fractional bandwidth of this simulated (measured) frequency-agile range reaches as high as $34.9 \%(36.0 \%)$. Moreover, the individual passbands are quite sharp. Their simulated (measured) -10-dB fractional bandwidths for these 
four junction capacitance values are $5.18 \%(4.19 \%), 4.53 \%$ (4.06\%), $3.15 \%(5.12 \%)$, and $2.40 \%(4.50 \%)$, respectively. As Fig. 9 demonstrates, quite good agreement between the simulated and measured results was obtained.

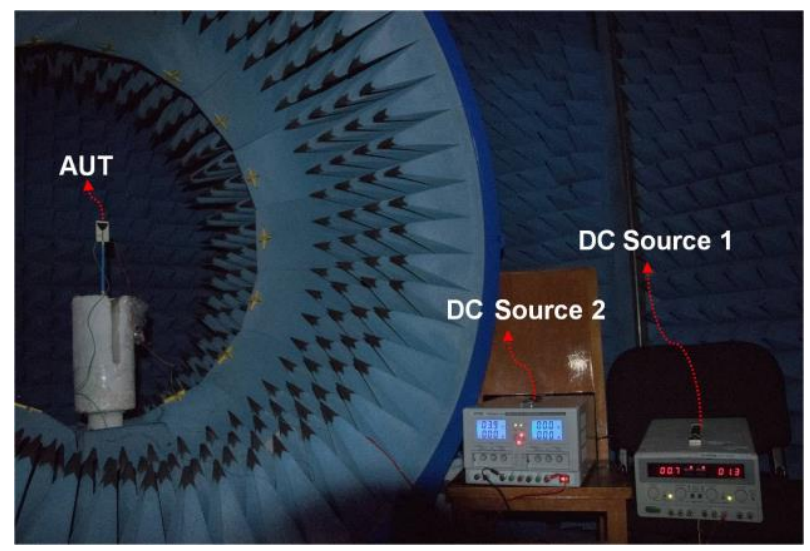

Fig. 10 Experimental setup used to measure the radiation performance of the filtenna. The antenna under test (AUT) and its two dc power sources are shown in the anechoic chamber.

The far-field radiation performance characteristics were investigated numerically and experimentally. The experiments were carried out in an anechoic chamber at UESTC, which is mainly based on an Agilent N5230A PNA-L VNA and a SATIMO passive measurement system [33]. The antenna under test (AUT) in the radiation pattern measurement system with its two DC power sources is depicted in Fig. 10. The PIN diode and the varactor diodes are fed with dc bias voltages provided by DC Source 1 and DC Source 2, respectively.

The simulated and measured normalized realized gain patterns at 2.4, 3.1, 4.3, $4.98 \mathrm{GHz}$ for the wideband state are presented and compared in Fig. 11. As expected, the E-plane and H-plane patterns are, respectively, basically monopole-like and omni-directional in all cases. Moreover, the filtenna has similar radiation patterns across the entire range of its wideband state $(2.35-4.98 \mathrm{GHz})$. Note that the radiation patterns witness a slight degradation at the highest frequencies near $5.0 \mathrm{GHz}$ as a result of the presence of higher order modes; this phenomenon has been extensively discussed previously [32].

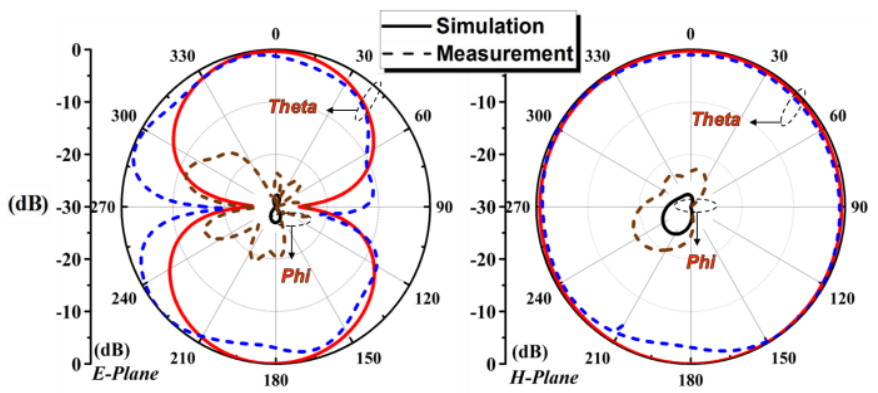

(a)

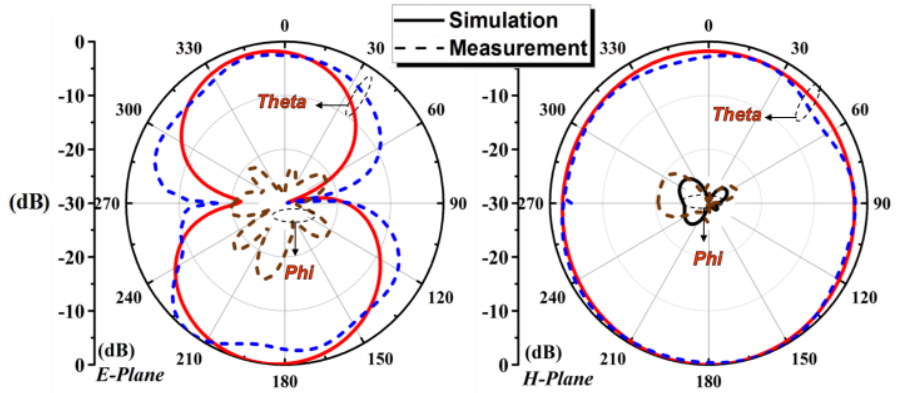

(b)

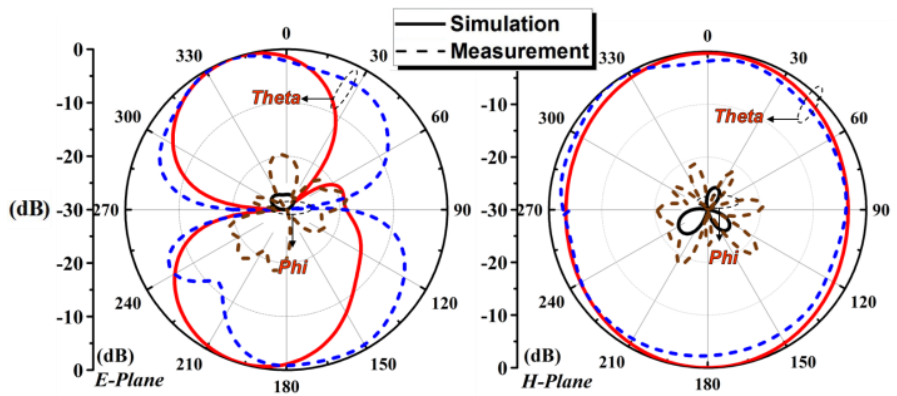

(c)

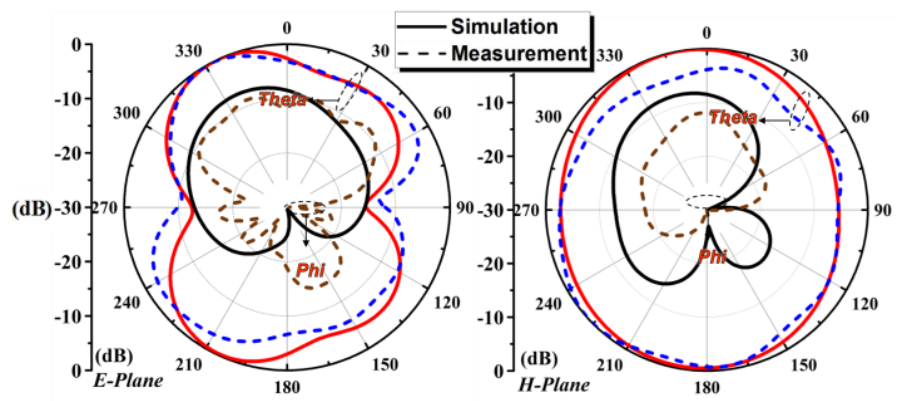

(d)

Fig. 11 Simulated and measured normalized realized gain patterns in the $E$-(ZOX) and $H$-(ZOY) planes at (a) 2.4, (b) 3.1 , (c) 4.3 , and (d) $4.98 \mathrm{GHz}$, when the filtenna is in its wideband state.

The far-field normalized realized gain patterns for the four narrowband states centered at $3.1,3.47,3.9$, and $4.3 \mathrm{GHz}$ are presented in Fig. 12. These frequencies correspond, respectively, to when each varactor was set to have the effective capacitance (reverse bias voltage): $0.1 \mathrm{pF}(20 \mathrm{~V}), 0.2 \mathrm{pF}(9 \mathrm{~V})$, $0.4 \mathrm{pF}(4.7 \mathrm{~V})$, and $1.0 \mathrm{pF}(2 \mathrm{~V})$. The radiation patterns in these instances are quite similar to those obtained when the filtenna was in its wideband state.

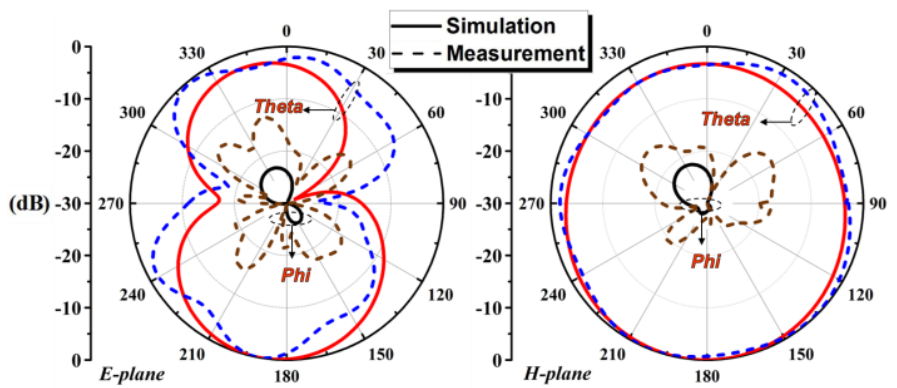

(a) 
COMPARISON OF THE FREQUENCY-RECONFIGURABLE FILTENNA PERFORMANCE CHARACTERISTICS WITH THOSE OF RELATED CR ANTENNAS REPORTED IN THE LITERATURE

\begin{tabular}{|c|c|c|c|c|c|c|c|c|c|}
\hline & $\begin{array}{c}\text { Dielectric } \\
\text { Constant }\end{array}$ & $\begin{array}{l}\text { Sensing } \\
\text { band } \\
(\mathrm{GHz})\end{array}$ & $\begin{array}{l}\text { Out-of-band } \\
\text { rejection in } \\
\text { sensing state }\end{array}$ & $\begin{array}{c}\text { Discrete / } \\
\text { Continuous }\end{array}$ & $\begin{array}{c}\text { Reconfigurable } \\
\text { communications band } \\
(\mathrm{GHz})\end{array}$ & $\begin{array}{l}\text { Frequency } \\
\text {-agile } \\
\text { fractional } \\
\text { bandwidth }\end{array}$ & $\begin{array}{l}\text { Tuning } \\
\text { ratio }\end{array}$ & $\begin{array}{c}\text { Ratio of } \\
\text { frequency-agile } \\
\text { to sensing } \\
\text { bandwidth }\end{array}$ & $\begin{array}{c}\text { The total electrical } \\
\text { size }\end{array}$ \\
\hline [4] & 4.6 & $2-8$ & $\mathrm{~N}$ & $\mathrm{D}$ & $5.1-5.9$ & -- & -- & -- & $0.4 \lambda_{L} \times 0.46 \lambda_{L}$ \\
\hline$[5]$ & 4.3 & $2-11$ & $\mathrm{~N}$ & $\mathrm{D}$ & $\begin{array}{l}2.1-2.6 / 3.6-4.6 / \\
2.8-3.4 / 4.9-5.8\end{array}$ & -- & -- & -- & $0.33 \lambda_{L} \times 0.33 \lambda_{L}$ \\
\hline$[6]$ & 2.2 & $3-11$ & $\mathrm{~N}$ & $\mathrm{D}$ & $3.2-4.3 / 4.15-5.1 / 4.8-5.7$ & -- & -- & -- & $0.455 \lambda_{L} \times 0.5 \lambda_{L}$ \\
\hline [7] & 4.4 & $2.5-12$ & $\mathrm{~N}$ & $\mathrm{D}$ & $\begin{array}{c}2.5-2.7 / 6.4-7 / \\
10.2-11.6 \\
\end{array}$ & -- & -- & -- & $0.28 \lambda_{L} \times 0.52 \lambda_{L}$ \\
\hline [8] & 2.33 & $0.83-2.16$ & $\mathrm{~N}$ & $\mathrm{D}$ & $\begin{array}{c}0.9-1 / 1.1-1.5 / \\
1.6-1.8 / 1.85-2.1\end{array}$ & -- & -- & -- & $0.664 \lambda_{L} \times 0.664 \lambda_{L}$ \\
\hline$[17]$ & 3.48 & $3.3-11$ & $\mathrm{~N}$ & $\mathrm{C}$ & $5-6$ & $18.2 \%$ & 1.2 & $13.00 \%$ & $0.396 \lambda_{L} \times 0.44 \lambda_{L}$ \\
\hline$[18]$ & 2.2 & $3.5-6$ & $\mathrm{~N}$ & $\mathrm{C}$ & $3.9-4.82$ & $21.1 \%$ & 1.24 & $36.40 \%$ & $0.291 \lambda_{L} \times 0.723 \lambda_{L}$ \\
\hline $\begin{array}{c}\text { This } \\
\text { work }\end{array}$ & 3.48 & $2.35-4.98$ & Y & $\mathrm{C}$ & $3.05-4.39$ & $36.0 \%$ & 1.44 & $50.95 \%$ & $0.235 \lambda_{\mathrm{L}} \times 0.392 \lambda_{\mathrm{L}}$ \\
\hline
\end{tabular}

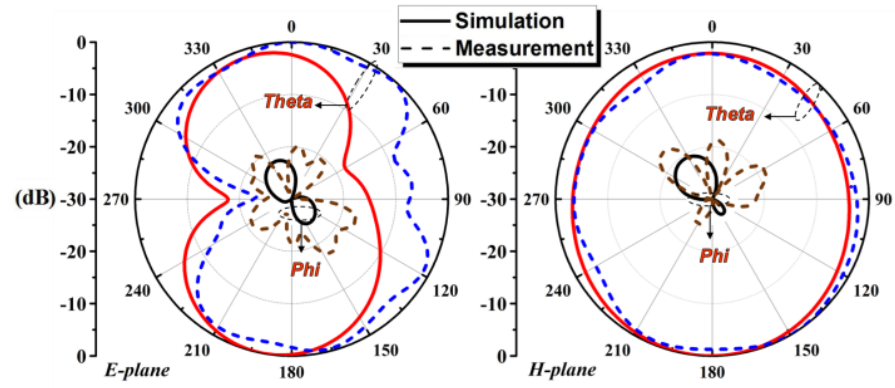

(b)

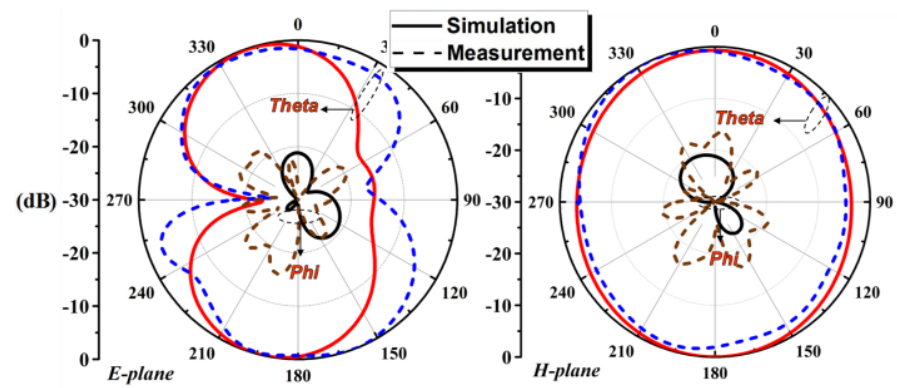

(c)

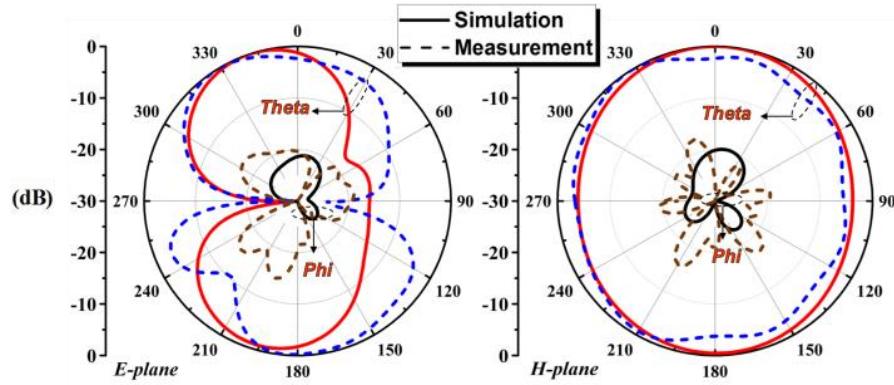

(d)

Fig. 12 Simulated and measured normalized radiation patterns in the $E$-(ZOX) and $H$-(ZOY) planes at (a) 3.1, (b) 3.47 , (c) 3.9 , and (d) $4.3 \mathrm{GHz}$, when the filtenna is in its narrowband state.

The simulated and measured realized gains of the proposed antenna for the wideband and narrowband states are shown in Fig. 13 as functions of the source frequency. For the wideband state, the simulated (measured) realized gain values range from $1.83(0.36)$ to $3.94(3.44) \mathrm{dBi}$ in the operational band. For the narrowband state, the simulated realized gain values were obtained at the resonance frequencies associated with each narrow passband filter response within the 3.12 to $4.44 \mathrm{GHz}$ interval obtained by continuously changing the junction capacitance of both varactors from 1.0 to $0.1 \mathrm{pF}$. They ranged from 1.59 to $4.08 \mathrm{dBi}$. On the other hand, the measured realized gain values were obtained at the resonance frequencies associated with each narrow passband filter response within the 3.05 to $4.39 \mathrm{GHz}$ interval obtained by continuously changing the DC voltage applied to each varactor from 2.0 to $20 \mathrm{~V}$. They vary from 1.4 to $2.55 \mathrm{dBi}$. Fig. 13 also gives the measured realized gain values as the functions of the source frequency at selected operational frequencies (junction effective capacitances): $3.1 \mathrm{GHz}(1.0 \mathrm{pF}), 3.47 \mathrm{GHz}(0.4 \mathrm{pF}), 3.9 \mathrm{GHz}$ $(0.2 \mathrm{pF})$, and $4.3 \mathrm{GHz}(0.1 \mathrm{pF})$. The observed differences between the simulated and measured realized gain values were deemed acceptable; they arose mainly from the usual unavoidable errors in the fabrication, assembly, and measurement processes.

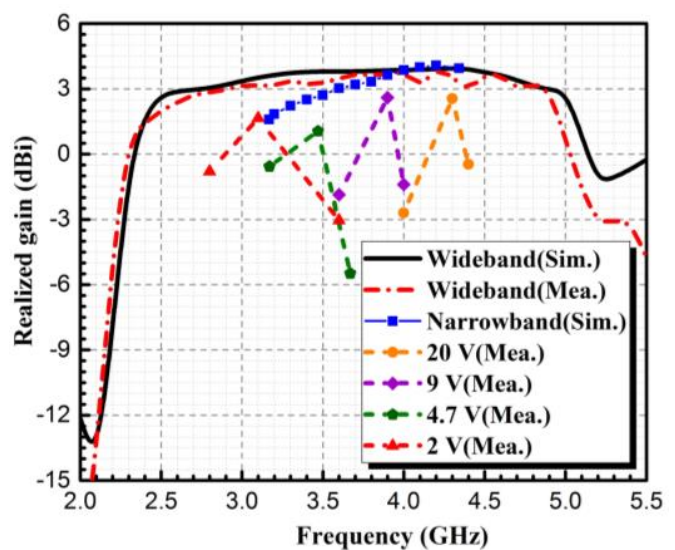

Fig. 13 Simulated and measured realized gains of the filtenna as functions of the source frequency.

The radiation efficiencies of the filtenna in its two states were also obtained. For the wideband state, they were $93.46 \%$, $96.68 \%$, and $95.39 \%$, respectively, at $2.5,3.5$, and $4.98 \mathrm{GHz}$. For the narrowband state, they were $67.16 \%, 81.64 \%$, and $80.92 \%$, respectively, at $3.47,4.1$, and $4.54 \mathrm{GHz}$. It is noted that the energy coupling in the narrowband state occurs mainly though the $\mathrm{C}$-shaped resonator, in which the two $1.75 \mathrm{ohm}$ 
varactor diodes were placed. Their presence causes the radiation efficiency of the filtenna to be lower in its narrowband state when compared to its wideband state. This was confirmed by taking the resistance of each varactor diode to be negligible $\left(\sim 10^{-10} \mathrm{ohm}\right)$ in the filtenna model. The simulated narrowband radiation efficiencies then reached much higher values: $81.40 \%$, $92.01 \%$, and $89.90 \%$, respectively, at $3.47,4.1$, and $4.54 \mathrm{GHz}$.

The measured results validate the simulated performance characteristics of the filtenna. In contrast to many of the previously reported reconfigurable CR filtennas, it not only has a simple and compact configuration, but it also possesses sharp out-of-band rejection properties in both its wideband and narrowband states. Table III provides a comparison to support these conclusions. The total electrical size, sensing bandwidth, communications switch type, communications bandwidth, tuning ratio, and ratio of the frequency-agile bandwidth to the wideband bandwidth are provided if they were available. Note that in order to make a fair comparison, the total electrical size reported corresponds to the lowest operational frequency within the $-10-\mathrm{dB}$ impedance bandwidth. While Table III highlights the compactness of our design, it also demonstrates that our filtenna possesses a much wider frequency-agile fractional bandwidth in its narrowband state and has a unique out-of-band-rejection performance in its wideband sensing state. Furthermore, the $50.95 \%$ value of the ratio of the frequency-agile bandwidth to the sensing (wideband) bandwidth is comparatively very high. We pointed out that this ratio value could reach as high as $100 \%$ by adjusting the size of E-shaped resonator, such as by adjusting the width of its stub $\left(\mathrm{L}_{5}\right)$.

\section{CONCLUSION}

A compact, frequency-reconfigurable filtenna with sharp out-of-band rejection in both sensing and continuously tunable communicating states for CR applications has been reported. A PIN diode is utilized to switch the operational state of the filtenna between sensing and communicating states. A square TLS is introduced to eliminate the notched dip within the sensing band. Two varactor diodes are inserted into $\mathrm{C}$-shaped narrowband resonator to shift the operational frequencies from 3.05 GHz to $4.39 \mathrm{GHz}$ at communicating state, yielding a wide tunable bandwidth $36.0 \%$. More importantly, the proposed filtenna has unique out-of-band rejection performance characteristics at the sensing state. Consequently, the proposed compact reconfigurable filtenna is expected to be suitable for a variety of cognitive radio systems in the near future.

\section{REFERENCES}

[1] Federal Communications Commission, "Spectrum Policy Task Force," Report of the spectrum efficiency working group. Technical Report, Nov. 2002.

[2] A. Petosa, "An overview of tuning techniques for frequency-agile antennas," IEEE Antennas and Propag. Mag., vol. 54, no. 5, pp. 271-296. Oct. 2012.

[3] M.-C. Tang, R. W. Ziolkowski, S. Xiao, M. Li, and J. Zhang, "Frequency-agile, efficient, near-field resonant parasitic monopole antenna," IEEE Trans. Antennas and Propag., vol. 62, no. 3, pp. 1479 1483, Mar. 2014.

[4] F. D. Dahalan, S. K. A. Rahim, M. R. Hamid, M. A. Rahman, M. Z. M. Nor, M. S. A. Rani, and P. S. Hall. "Frequency-reconfigurable
Archimedean spiral antenna," IEEE Antennas and Wireless Propag. Lett., vol. 12, pp. 1504-1507, 2013.

[5] T. Aboufoul, A. Alomainy, and C. Parini, "Reconfigurable UWB monopole antenna for cognitive radio applications using GaAs FET switches," IEEE Antennas and Wireless Propag. Lett., vol. 11, pp. 392-394, 2012.

[6] Y. Tawk, J. Costantine, S. Hemmady, G. Balakrishnan, K. Avery, and C. G. Christodoulou, "Demonstration of a cognitive radio front end using an optically pumped reconfigurable antenna system (OPRAS)," IEEE Trans. Antennas and Propag., vol. 60, no. 2, pp. 1075-1083, Feb. 2012.

[7] N. V. Soumya and P. R. Anurenjan, "Swithced-band Vivaldi antenna with hexagonal slots for cognitive radio applications," 2016 IEEE Annual India Conference (INDICON), Bangalore, India, 2016, pp. 1-4.

[8] L. Ge and K. M. Luk, "Band-reconfigurable unidirectional antenna: A simple, efficient, magneto-electric antenna for cognitive radio applications," IEEE Antennas and Propag. Mag., vol. 58, no. 2, pp. 18-27, Apr. 2016.

[9] R. Hussain and M. S. Sharawi, "A cognitive radio reconfigurable MIMO and sensing antenna system," IEEE Antennas and Wireless Propag. Lett., vol. 14, pp. 257-260, 2015.

[10] A. Mansoul, F. Ghanem, M. R. Hamid, and M. Trabelsi, "A selective frequency-reconfigurable antenna for cognitive radio applications," IEEE Antennas and Wireless Propag. Lett., vol. 13, pp. 515-518, 2014.

[11] M. Bitchikh and F. Ghanem, "A three-resolution UWB frequency-reconfigurable antipodal Vivaldi antenna for cognitive radios," in Proc. $8^{\text {th }}$ European Conference on Antennas and Propagation (EuCAP 2014), The Hague, Netherlands, 2014, pp. 3665-3668.

[12] F. Ghanem, and A. Mansoul, "An UWB to four sub-bands frequency-reconfigurable CPW-fed disc monopole antenna," in Proc. IEEE Antennas and Propagation Society International Symposium (APSURSI), Orlando, FL, 2013, pp. 690-691.

[13] B. P. Chacko, G. Augustin, and T. A. Denidni, "Electronically reconfigurable uniplanar antenna with polarization diversity for cognitive radio applications," IEEE Antennas and Wireless Propag. Lett., vol. 14, pp. 213-216, 2015.

[14] M. Zamudio, Y. Tawk, J. Costantine, J. Kim, and C. G. Christodoulou, "Integrated cognitive radio antenna using reconfigurable band pass filters," in Proc. $5^{\text {th }}$ European Conference on Antennas and Propagation (EuCAP2011), Rome, Italy, 2011, pp. 2108-2112.

[15] N. Kumar, P. A. Raju, and S. Kumar Behera, "Frequency-reconfigurable microstrip antenna for cognitive radio applications," in Proc. 2015 International Conference on Communications and Signal Processing (ICCSP'2015), Melmaruvathur, India, 2015, pp. 0370-0373.

[16] P. - Y. Qin, Y. J. Guo, and F. Wei, "Frequency agile monopole antenna using a reconfigurable bandpass filter," in Proc. 2014 IEEE Antennas and Propagation Society International Symposium (APSURSI), Memphis, TN, 2014, pp. 1250-1251

[17] E. Erfani, J. Nourinia, C. Ghobadi, M. Niroo-Jazi, and T. A. Denidni, "Design and implementation of an integrated UWB/reconfigurable-slot antenna for cognitive radio applications," IEEE Antennas and Wireless Propag. Lett., vol. 11, pp. 77-80, 2012.

[18] P.-Y. Qin, F. Wei, Y. and J. Guo, "A wideband-to-narrowband tunable antenna using a reconfigurable filter," IEEE Trans. Antennas Propag., vol. 63, no. 5, pp. 2282-2285, May 2015.

[19] J. Zuo, X. Chen, G. Han, L. Li, and W. Zhang, "An integrated approach to RF antenna-filter co-design," IEEE Antennas Wirel. Propag. Lett., vol. 8, pp. 141-144, 2009.

[20] Z. H. Jiang, and D. H. Werner, "A compact, wideband circularly polarized co-designed filtering antenna and its application for wearable devices with low SAR," IEEE Trans. Antennas Propag., vol.63, no. 9, pp. 3808-3818, Sep. 2015.

[21] M.-C. Tang, Y. Chen, and R. W. Ziolkowski, "Experimentally validated, planar, wideband, electrically small, monopole filtennas based on capacitively loaded loop resonators," IEEE Trans. Antennas and Propag., vol. 64 , no. 8, pp. 3353-3360, Aug. 2016.

[22] ANSYS/ANSOFT High Frequency Structure Simulation (HFSS), ver. 13.0, ANSYS Corp. [Online]. Available at www.ansoft.com.

[23] J. S. Hong and M. J. Lancaster, Microstrip filters for RF/microwave applications, New York: Wiley, 2001.

[24] K. Srisathit, and W. Surakumpontorn, "Wideband microstrip bandpass filter based on modified parallel-coupled line topology," in Proc. 2010 $10^{\text {th }}$ International Symposium on Communications and Information Technologies, Tokyo, Japan, 2010, pp. 45-48

[25] S. Sun, L. Zhu, "Multimode-resonator-based bandpass filters," IEEE Microw. Mag., vol. 10, no. 2, pp. 88-98, Apr. 2009. 
[26] S. Sun, L. Zhu, "Capacitive-ended interdigital coupled lines for UWB bandpass filters with improved out-of-band performances," IEEE Microw. Wireless Compon. Lett., vol. 16, no. 8, pp. 440-442, Aug. 2006.

[27] S. Sun, L. Zhu, "Periodically nonuniform coupled microstrip-line filters with harmonic suppression using transmission zero reallocation," IEEE Trans. Microw. Theory Tech., vol.53, no. 5, May 2005.

[28] [Online]. Available http:// cdn.macom.com/ datasheets/MA4GP907.pdf

[29] [Online]. Available http://ams.areoflex.com/Metelics/pdfles/MGV_ Series_Hyperabrupt_A 17041.pdf

[30] F. S. Mahmud, M. S. Razalli, W. F. Hoon, S. Z. Ibrahim, and N. B. M. Hashim, "Tuneable dual-mode narrowband bandstop filter using loaded microstrip resonator," in Proc. $20163^{\text {rd }}$ International Conference on Electronic Design (ICED 2016), Phuket, Thailand, 2016: 80-85

[31] C.-C. Lin, P. Jin, and R. W. Ziolkowski, "Single, dual and tri-band-notched Ultrawideband (UWB) antennas using capacitively loaded loop (CLL) resonators," IEEE Trans. Antennas and Propag., vol. 60, no. 1, pp.102-109, Jan. 2012.

[32] M.-C. Tang, R. W. Ziolkowski, and S. Xiao, "Compact hyper-band printed slot antenna with stable radiation properties," IEEE Trans. Antennas Propag., vol. 62, no. 6, pp. 2962-2969, Jun. 2014.

[33] StarLab Version D User Guide 1.0, Reference: TD. 224.1.08. SATF. A, SATIMO Corporations 2008. 